Check for updates

Cite this: Phys. Chem. Chem. Phys., 2020, 22, 18824

Received 20th June 2019

Accepted 21st August 2019

DOI: $10.1039 / c 9 c p 03473 k$

rsc.li/pccp

\title{
Identification of the key steps in the self-assembly of homogeneous gold metal nanoparticles produced using inverse micelles $\dagger$
}

\author{
Donato Decarolis, ${ }^{\text {ab }}$ Yaroslav Odarchenko, (D) ab Jennifer J. Herbert, ${ }^{\text {abc }}$ \\ Chengwu Qiu, $^{\mathrm{ab}}$ Alessandro Longo ${ }^{\mathrm{cd}}$ and Andrew M. Beale (D) *ab
}

\begin{abstract}
The self-assembly of gold nanoparticles (Au NPs) using polymer-encapsulated inverse micelles was studied using a set of advanced X-ray techniques (i.e. XAFS, SAXS) in addition to DLS, UV-vis spectroscopy and TEM. Importantly the combination of these techniques with the inverse micelle approach affords us detailed insight and to rationalize the evolving molecular chemistry and how this drives the formation of the Au NPs. We observe that the mechanism comprises three key steps: an initial fast reduction of molecular Au(II) species to molecular $\mathrm{Au}(\mathrm{I}) \mathrm{Cl}$; the latter species are often very unstable during the self-assembly process. This is followed by a gradual reduction of these molecular $\mathrm{Au}(\mathrm{l})$ species and the formation of sub-nanometric $\mathrm{Au}$ clusters which coalesce into nanoparticles. It was also found that addition of small amounts of $\mathrm{HCl}$ can accelerate the formation of the Au clusters (the second phase) without affecting the final particle size or its particle size distribution. These findings would help us to understand the reaction mechanism of Au NP formation as well as providing insights into how NP properties could be further tailored for a wide range of practical applications.
\end{abstract}

\section{Introduction}

Metal nanoparticles (NPs) can be used in a variety of applications ranging from catalysis to sensors, antibacterials and drug delivery. ${ }^{1-4}$ In order to prepare a metal nanoparticle, various methods have been developed. ${ }^{5,6}$ Synthesis methods can be divided into top-down methods (NPs are removed from 'bulk' material) and bottom-up (atoms assemble NPs). ${ }^{5,6}$ Whereas top-down approaches, such as e-beam lithography ${ }^{7}$ or thermal evaporation, ${ }^{8}$ allow NP production with regular physical attributes (e.g. size and shape). It is generally not viable for large scale applications, for example, to manufacture heterogeneous catalysts, due to the time required to produce the material, expensive instrumentation and the fact that it only allows for the formation of $1 \mathrm{D}$ and $2 \mathrm{D}$ supported NP arrays ${ }^{9}$ and therefore is mainly suitable to fabricate model systems. The bottom-up approach, more commonly reported as a method to prepare

\footnotetext{
${ }^{a}$ Department of Chemistry, University College London, 20 Gordon Street, London, WC1H OAJ, UK. E-mail: andrew.beale@ucl.ac.uk

${ }^{b}$ Research Complex at Harwell (RCaH), Harwell, Didcot, Oxfordshire OX11 OFA, UK ${ }^{c}$ Netherlands Organisation for Scientific Research (NWO), DUBBLE CRG@ESRF, Grenoble 38042, France

${ }^{d}$ Istituto per lo Studio dei Materiali Nanostrutturati, Sezione di Palermo, Consiglio Nazionale delle Ricerche, Via La Malfa, 153, I-90146 Palermo, Italy $\dagger$ Electronic supplementary information (ESI) available. See DOI: 10.1039/ c9cp $03473 \mathrm{k}$
}

NPs, involves the reduction of metal ions in solution and a subsequent agglomeration. ${ }^{5,10-13}$ Bottom-up methods include templating, ${ }^{5}$ chemical,${ }^{10}$ electrochemical ${ }^{11}$ and sonochemical ${ }^{12}$ reduction, with a common occurrence in these types of synthesis being the presence of a capping agent, often a surfactant, in order to restrict the NPs growth to the desired size and shape. The main disadvantage of these bottom-up approaches is that they rarely produce homogeneous NPs, often resulting in a broad particle size distribution and the average particle size may vary from batch to batch. This uncertainty in the NP size makes it difficult to truly understand the impact of NPs size on their properties e.g. catalytic activity ${ }^{14,15}$ or for other applications such as colorimetric sensing. ${ }^{3}$ Despite these drawbacks, bottom-up methods are used for industrial applications, such as production of the fixed bed heterogeneous catalysts, mainly for being relatively fast and straightforward and resulting in lower cost compared to top-down methods. Therefore, improving the synthesis methods in order to produce NPs with a particle size distribution as narrow as possible is paramount for the improvement in the understanding of NP properties.

The fabrication of monodisperse NPs using bottom-up approaches, however, is not trivial and is hampered by the lack of detailed knowledge of all the steps in the self-assembly process. ${ }^{5}$ Various studies have been performed in order to understand the evolution of colloidal solution from the metal 
ions to NPs in different systems, such as $\mathrm{Au},{ }^{10} \mathrm{Fe}_{2} \mathrm{O}_{3},{ }^{16} \mathrm{Ag},{ }^{17}$ and several NPs' growth modes including Ostwald ripening, fusion, coalescence, condensation and coagulation, and combinations of these modes, thoroughly described in a review article from Thanh et al. ${ }^{18}$ Despite similarities in the fabrication mechanisms, the NP growth shows some dependence on the synthesis method. ${ }^{10,19}$ Perhaps the best known self-assembly study concerns the work of Polte et al. who studied the mechanism of $\mathrm{Au}$ NPs formation in the commonly used citrate method and showed that the combination of Small-angle X-ray Scattering (SAXS) and X-ray Absorption Near Edge Structure (XANES) analysis is able to reveal insight into the growth of metal nanoparticles during synthesis. ${ }^{10}$ The authors concluded that NP growth goes through four different phases:

- fast initial formation of small gold nuclei comprising a few atoms;

- coalescence of these nuclei into a nanoparticle;

- slow growth of the nanoparticle sustained by an ongoing reduction of the precursor;

- fast reduction ending with the complete consumption of the precursor species.

The second step (coalescence of the nuclei) has been identified as a key step influencing the polydispersity of the nanoparticles. ${ }^{10}$ However it is challenging to control multiple factors such as reagents mixing and $\mathrm{pH}$ of the citrate solution acting as reducing agent and stabiliser. On the other hand, a study from Sakai et al. shows that in the case of a block copolymer (poly(ethylene oxide)poly(propylene oxide)-poly(ethylene oxide), also known as PEOPPO-PEO) the NPs can be grown in a single step, due to the presence of the polymer acting both as a stabilizer as well as a reductant. ${ }^{19}$ Here the presumed mechanism of gold NPs formation is proposed as below:

- reduction of the metal ions facilitated by the polymer to form clusters (i.e. structures containing a small number of atoms);

- adsorption of the polymer onto the cluster surface and a (further) reduction of $\left[\mathrm{AuCl}_{4}\right]^{-}$ions adsorbed on the surface of the clusters;

- growth of the NP through a repeat of step 2 until stabilisation.

In this process the polymer plays an important role in determining the particle size resulting from the synthesis. This is due to the dual attribute of the polymer as reductant and stabilizer, which varies the reaction activity of $\left[\mathrm{AuCl}_{4}\right]^{-}$based on the polymer molecular weight. ${ }^{19}$ By comparing the citrate ${ }^{10}$ and copolymer-assisted ${ }^{19}$ methods one can conclude that the reduction of metal ions into a cluster and the coalescence of the clusters are two common steps during metal NP synthesis. However, there are also key differences in the mechanism for gold NP growth, the reasons for which could be attributable to the different stabilizer (citrate in one case and block copolymer in the other) that could alter the pathway of the reduction process. Ideally, a detailed investigation is required to determine the suggested mechanism for Au NP synthesis using the PEOPPO-PEO based method, however considering it is a "single pot" synthesis procedure it could be extremely challenging to unravel the many chemical processes that lead to the formation of the $\mathrm{Au}$ NPs. ${ }^{19}$

Among the bottom-up methods, polymer-encapsulated inverse micelle synthesis, introduced by Spatz et al., can be used to prepare, in a relatively quick and reproducible way, uniform monodisperse metal NPs which can be conveniently loaded onto a variety of supports, e.g. refractive oxide powders. ${ }^{20-22}$ This advanced synthesis method mimics water-in-oil systems by employing precursor-filled micelles formed by PS- $b$-P2VP diblock-copolymer with low polydispersity index (PDI) in organic solvent, that allows for full encapsulation of all metal ions and inhibits the formation of sub-species. ${ }^{22}$ Another advantage of this approach includes self-assembly into regular arrays with controlled inter-particle distance which, when deposited on a flat substrate, can improve the properties of the final material e.g. minimises the interactions between NPs and prevents sintering during heat treatment. ${ }^{22,23}$ The size of the deposited NPs and their density (e.g. inter-particle distance) can be conveniently controlled by altering the length of shell- (PS) or core-forming (P2VP) copolymer blocks. ${ }^{23}$ Despite the multiple reports where the polymer-based method has been shown to produce well-defined metal NPs with suitably small particle size distributions, ${ }^{20,22-25}$ to the best of our knowledge no detailed studies have been performed to understand the mechanism of metal NP formation. As such important questions remain regarding the time required to complete the self-assembly and reduction processes, and the step-by-step pathway to self-assemble metal ions into Au NPs and whether/ how such methods can be exploited to prepare multicomponent/ multi-layered structures such as core-shell multi-metallics. Although the use of inverse micelles consisting in the encapsulation of Au species inside AOT (dioctyl sodium sulfosuccinate) liquid crystal has been explored in the literature, ${ }^{8,26}$ this approach would not have made possible the in situ observation of the formation mechanism of the gold NPs, compared to the solvent based method here employed.

In situ X-ray Absorption Fine Structure (XAFS) is a powerful method to follow the changes in the electronic structure of metals during the reduction process..$^{27,28}$ In particular, XANES is used to observe changes in oxidation state of the Au species, whereas Extended X-ray Absorption Fine Structure (EXAFS) technique can monitor the formation of $\mathrm{Au}$ clusters through detection of the Au-Au scattering pair evolution. ${ }^{10,27}$ Whereas XAFS reveals information on the local environment of the gold atoms (i.e. the short-range order) in the micelles, SAXS is sensitive (in a size range of 1 to $100 \mathrm{~nm}$ ) to the changes in the electron density fluctuations and is more suitable to probe the micelles' core where gold clusters formation occurs. ${ }^{10,17}$ Thus, a combination of these two X-ray techniques with the use of other more commonly available experimental methods such as dynamic light scattering (DLS), ultraviolet-visible spectroscopy (UV-vis) and transmission electron microscopy (TEM), allows to understand the chemical and physical processes happening during the formation of the metal NPs.

This work is focused on the investigation of gold NP formation during polymer-encapsulated inverse micelle synthesis using in situ X-ray spectroscopy and scattering. The inverse 
micelle method, by offering tight control over the NPs' size, allows a more detailed mechanistic insight into the process of homogeneous NP formation. Key to this study is the avoidance of 'fast' reducing agents such as $\mathrm{NaBH}_{4}{ }^{29}$ or citrate, ${ }^{10}$ that can often result in uncontrolled particle growth and a large particle size distribution, hindering our attempts at a detailed understanding of the selfassembly process. ${ }^{30}$ By modifying the inverse micelle method developed by Spatz et al., using a slower reductant compared to the commonly used hydrazine, it was possible to observe the presence of $\mathrm{Au}(\mathrm{I})$ species, previously supposed to be an intermediate in the Au nanoparticle formation but hardly detected due to their inherent instability. Furthermore, it was observed that the addition of small quantities of $\mathrm{HCl}$ during the synthesis appear to catalyse the formation of Au nanoclusters, most probably due to the formation of in situ $\mathrm{N}_{2} \mathrm{H}_{4}$. The insight obtained using these advanced characterization methods in combination with the inverse micelle synthesis allowed us to identify the key stages in the self-assembly process that are useful when trying to optimize NPs for a wide range of practical applications.

\section{Experimental section}

\subsection{Nanoparticles synthesis}

Gold nanoparticle solutions were prepared using the polymerencapsulated inverse micelles method. ${ }^{20}$ Poly(styrene- $b$-2-vinyl pyridine) (PS- $b$-P2VP) block-copolymer P18226-S2VP was purchased from the Polymer Source Inc (electronic grade purity). The metal salt used was chloroauric acid $\left(\mathrm{HAuCl}_{4}\right)$ (Aldrich $99.99999 \%$ trace metal basis). $p$-Toluene sulfonyl hydrazide ( $p$-tosyl hydrazide) (Aldrich 97\%) was used as the reducing agent, as an alternative to the more commonly used toxic and explosive hydrazine. ${ }^{31}$ All reagents were used as received. $0.5 \mathrm{wt} \%$ polymer $\left(c=5 \mathrm{mg} \mathrm{ml}{ }^{-1}\right)$ solution was prepared in $25 \mathrm{ml}$ of toluene (Aldrich 99.9\%) and left to stir for 3 days in order to allow the polymer to dissolve and homogenize. After 3 days, $0.021 \mathrm{~g}$ of the metal precursor was added to the solution and left to allow the metal salt to incorporate inside the micelles. The metal atoms to pyridine ratio (metal loading) was fixed at 0.3 . Before the measurements $10 \mathrm{ml}$ of metal loaded polymer solution was put in a glass vial with a $10 \mathrm{ml}$ of a solution of $p$-tosyl hydrazide. To compare the effect of the $\mathrm{HCl}$, used in literature to remove the excess of hydrazide, ${ }^{20}$ on the metal reduction inside the polymer micelle, $50 \mu \mathrm{l}$ of $\mathrm{HCl}$ (ACS reagent $37 \%$, Fluka) was added to the solution after $\sim 30 \mathrm{~s}$ and left to stir. Table 1 summarises a list of the samples used in this work.

\subsection{In situ small-angle X-ray scattering (SAXS)}

In situ Small-angle X-ray scattering (SAXS) measurements were conducted on the DUBBLE beamline (BM26A) of the European

Table 1 Samples list

\begin{tabular}{lllll}
\hline Sample & Polymer & $M_{\mathrm{n}} \times 10^{3}, \mathrm{~g} \mathrm{~mol}^{-1}$ & $\mathrm{PDI}^{a}$ & HCl volume $(\mu \mathrm{l})$ \\
\hline A & P18226-S2VP & $\mathrm{PS}(30.0)-b-\mathrm{P} 2 \mathrm{VP}(8.5)$ & 1.06 & 0 \\
A-HCl & P18226-S2VP & $\mathrm{PS}(30.0)-b$-P2VP(8.5) & 1.06 & 50
\end{tabular}

${ }^{a}$ PDI - polydispersity index.
Synchrotron Radiation Facility (ESRF) using X-ray photons of $11.850 \mathrm{keV}$. The 2D SAXS images were recorded in transmission mode over a period of 3 min using the Mar CCD 165 detector with a pixel size of $80 \mu \mathrm{m}$. The modulus of the scattering vector $\mathbf{q}=4 \pi \sin \theta /$ $\lambda$, where $\theta$ is the Bragg angle and $\lambda$ is the wavelength, was calibrated using silver behenate. The sample to detector distance was approximately $2 \mathrm{~m}$, resulting in a $q$ range spanning 0.016 and $0.4 \mathrm{~nm}^{-1}$.

For the sample measurement, an aliquot of each solution, taken at different reduction times $(0,0.3,1.25,2.25,6.25 \mathrm{~h})$, was loaded in $1 \mathrm{ml}$ quartz capillaries of $1 \mathrm{~mm}$ diameter. The data were corrected for the detector dark field and background scattering from the capillary with polymer and solvent only. The data reduction was performed using Fit2D and 1D curves were fitted using SANS Analysis package for the Igor Pro software. ${ }^{32}$

2.2.1 SAXS analysis. Scattering intensity of the Au NPS solution reads as follows

$$
I(q)=A \cdot \int_{0}^{\infty} F(q) f(R) \mathrm{d} R
$$

where $A$ is the scaling parameter, $F(q)$ - NP form factor, $f(R)$ NPs distribution function.

The Schultz-Zimm distribution function was chosen, that can account for the asymmetrical NPs size distribution. Also this model was successfully used in the past to reveal the mechanism of the Au NPs formation in the classical citrate method. ${ }^{10}$ The Schultz-Zimm distribution can be written as ${ }^{33}$

$$
f(R)=\left(\frac{z+1}{R_{\mathrm{a}}}\right)^{z+1} R^{z} \exp \left[-\left(\frac{z+1}{R_{\mathrm{a}}} R\right)\right] \frac{1}{\Gamma(z+1)}
$$

where $R_{\mathrm{a}}$ - is the mean radius, $z$ - is a parameter related to the polydispersity $\left(\rho=\sigma / R_{\mathrm{a}}\right)$ by $\rho=1 /(z+1)^{0.5}$ where $\sigma$ is the rootmean-square deviation from the mean size.

NP (P2VP core of the core-shell polymer micelle) is considered to be a hard (uniform density) sphere with the radius $R$ and volume $V$. This approximation is supported by the electron microscopy data that is discussed later in the paper. NP's form factor is given by the Rayleigh formula ${ }^{34}$

$$
F(q)=(\Delta \rho)^{2} \cdot V^{2}\left[3 \frac{\sin (q R)-q R \cos (q R)}{(q R)^{3}}\right]^{2}
$$

where $\Delta \rho$ - density difference between the particle and the medium, $q$ - modulus of the scattering vector.

\subsection{In situ X-ray absorption fine structure (XAFS)}

Extended X-ray absorption fine structure (EXAFS) and X-ray Absorption Near Edge Structure (XANES) were measured on the DUBBLE beamline (BM26A) of the European Synchrotron Radiation Facility (ESRF) using $\mathrm{Au} \mathrm{L}_{\mathrm{III}}$ edge (11.91 keV) in fluorescence mode using a 9-element monolithic Ge detector (Ortec), with the sample holder mounted at $45^{\circ}$ with the respect to the incident beam in order to maximize the fluorescence yield. An aliquot of each solution, taken at different times during the reduction process ( 0 and $2.25 \mathrm{~h}$ ), was loaded in $1 \mathrm{ml}$ quartz capillary and mounted on the sample holder. Each XAFS spectrum was acquired over a period of $\sim 10 \mathrm{~min}$ and repeated a further 3 times for each sample measured. The 
reference data used for XANES analysis were obtained by the author during previous XAFS experiment at the B18 beamline of Diamond Light Source (DLS) using Au $\mathrm{L}_{\mathrm{III}}$ edge in transmission mode on a solution of $\mathrm{AuCl}_{3}$ in water and on an $\mathrm{Au}$ foil for $\mathrm{Au}(\mathrm{III})$ and $\mathrm{Au}^{0}$ reference, respectively.

2.3.1 EXAFS analysis. EXAFS analysis was performed using Athena and Artemis from the Demeter IFEFFIT package. ${ }^{35}$ The FEFF6 $^{36}$ code was used to construct theoretical EXAFS signals that included single-scattering contributions from atomic shells through the nearest neighbours in the chlorine-bridged dimer, as well as the face-centred cubic (FCC) structure of Au. The $k$-range used for the fitting spanned 2.5 to $10.3 \AA^{-1}$ and the $r$-range from 1 to $3.7 \AA$. The path degeneracy was allowed to vary in order to account the size effects that cause surface atoms to be less coordinated than those in the particle interior. The amplitude reduction factor $\left({S_{0}}^{2}\right)$ was fixed at 0.860, as obtained from the fitting of the bulk Au foil.

\subsection{Transmission electron microscopy (TEM)}

Transmission Electron Microscopy (TEM) was measured using JEOL: JEM-2100 operating at $200 \mathrm{keV}$ using a $\mathrm{LaB}_{6}$ filament, after deposition through drop-casting on a carbon film supported by a 300 mesh copper TEM grid. Sample A-HCL solution before and after the reduction treatment were analysed. Due to a small scattering contrast of the block copolymer compared to the carbon film renders it impossible to obtain information regarding the state of the micelles before the addition of the Au precursor.

\subsection{UV-vis spectroscopy}

UV-vis spectroscopy measurements were taken in transmission mode, using a Shimadzu UV-1800 spectrometer for sample A and an Agilent Cary series for sample B, with the samples loaded into a cuvette with $5 \mathrm{~mm}$ pathlength to determine the position of the Au plasmon peak. A toluene solution with the polymer micelles was used as a background in order to remove any interference to the plasmon peak. Each spectrum was acquired using a total of 5 min per scan.

\subsection{Dynamic light scattering (DLS)}

Dynamic light scattering (DLS) measurements were performed on samples reduced over different times using Zetasizer nano ZS from Malvern. The incident laser wavelength used in the setup was $532 \mathrm{~nm}$ and the refractive index was derived from polystyrene $(\eta=1.5)$. Micelles containing solutions were loaded into a cuvette with $5 \mathrm{~mm}$ pathlength.

\section{Results and discussion}

\subsection{DLS}

With the help of DLS one can observe how the micellar system in its entirety responds during the reduction of metal ions through the impact this has on the micelles' hydrodynamic diameter. DLS plots, including the weighted average micelle radius obtained from the DLS measurement for sample A during reduction are shown in Fig. 1. The mean size of the micelles before reduction appears to be around $60 \mathrm{~nm}$ which increases to $70 \mathrm{~nm}$ in $0.1 \mathrm{~h}$ after addition of the reducing agent. As can be seen on Fig. 1 the swelling of the metal-containing polymer particles continues and results in almost doubling of the mean micelle size. A similar effect is observed in sample A- $\mathrm{HCl}$ when $\mathrm{HCl}$ is added during the reduction process, where the micelles size increases from 60 to $100 \mathrm{~nm}$ with the reduction time.

We propose that the observed increase of the micelle size is a consequence of the reduction of the metal salt. The reduction reaction of $\left[\mathrm{AuCl}_{4}\right]^{-}$with $\mathrm{H}_{2}$, produced by the $p$-tosyl hydrazide decomposition, to $\mathrm{Au}(\mathrm{I})$ is thought to occur as follows:

$$
\mathrm{H}\left[\mathrm{AuCl}_{4}\right]^{-}+\mathrm{H}_{2} \rightarrow \mathrm{AuCl}+3 \mathrm{HCl}
$$

and the reduction of $\mathrm{Au}(\mathrm{I})$ to form metallic $\mathrm{Au}^{0}$

$$
2 \mathrm{AuCl}+\mathrm{H}_{2} \rightarrow 2 \mathrm{Au}+2 \mathrm{HCl}
$$

which can be summarised as follows

$$
2 \mathrm{H}\left[\mathrm{AuCl}_{4}\right]^{-}+3 \mathrm{H}_{2} \rightarrow 2 \mathrm{Au}^{0}+8 \mathrm{HCl}
$$

In eqn (6) there is doubling in the number of moles formed in the reaction leading to an increased volume from within the micelles. Furthermore, this results in an increased entropy (and subsequent free-energy change) for the formation of $\mathrm{Au}^{0}$ species and its further agglomeration into nanoparticles. Another factor which may cause the swelling of micelles is the electrostatic strain caused by the formation of small Au clusters.

DLS studies on the similar systems have been reported before including copolymer- and surfactant-based inverse micelles, ${ }^{37-39}$ colloidal gold and polystyrene nanoparticles, ${ }^{40,41}$ and inverse micelles filled with $\mathrm{Au}$ NPs. ${ }^{42,43}$ In the most relevant study Mössmer et al., ${ }^{42}$ using PS- $b$-P2VP with block lengths comparable to sample A, observed a mean radius of $28 \mathrm{~nm}(56 \mathrm{~nm}$ diameter) for the micelles filled with $\mathrm{H}\left[\mathrm{AuCl}_{4}\right]$, which is similar to the size of $60 \mathrm{~nm}$ measured for sample A before reduction. In their study, the micelles' size for fully reduced Au NPs was reported to increase to $30 \mathrm{~nm}$ (60 $\mathrm{nm}$ diameter), compared to the $110 \mathrm{~nm}$ diameter observed here for sample $\mathrm{A}$ at the end of the reduction process. ${ }^{42}$ This discrepancy could be due to the different reductant used: hydrazine ${ }^{42}$ versus the much bulkier $p$-tosyl hydrazide used here. Also the authors did not perform a time-resolved study and thus the intermediate stages of the reduction process were not seen, ${ }^{42}$ whereas our DLS measurements as a function of time allows us to follow the swelling of the micelles as the reduction process is taking place (Fig. 1). It has to be noted that DLS is only capable of measuring the total diameter of the core-shell polymer micelle, consisting of the PS core and P2VP shell based on the miscibility of copolymer blocks, and does not allow for probing the local structural changes concerning the Au NPs of primary interest. ${ }^{42}$ Therefore, the combination of microscopic and spectroscopic methods have also been employed to obtain information regarding the local environment of the gold loaded P2VP core and the role of $\mathrm{HCl}$ in the reduction process.

\subsection{UV-vis spectroscopy}

UV-vis spectroscopy was employed to follow Au species evolution in solution during the reduction process. The UV-vis 

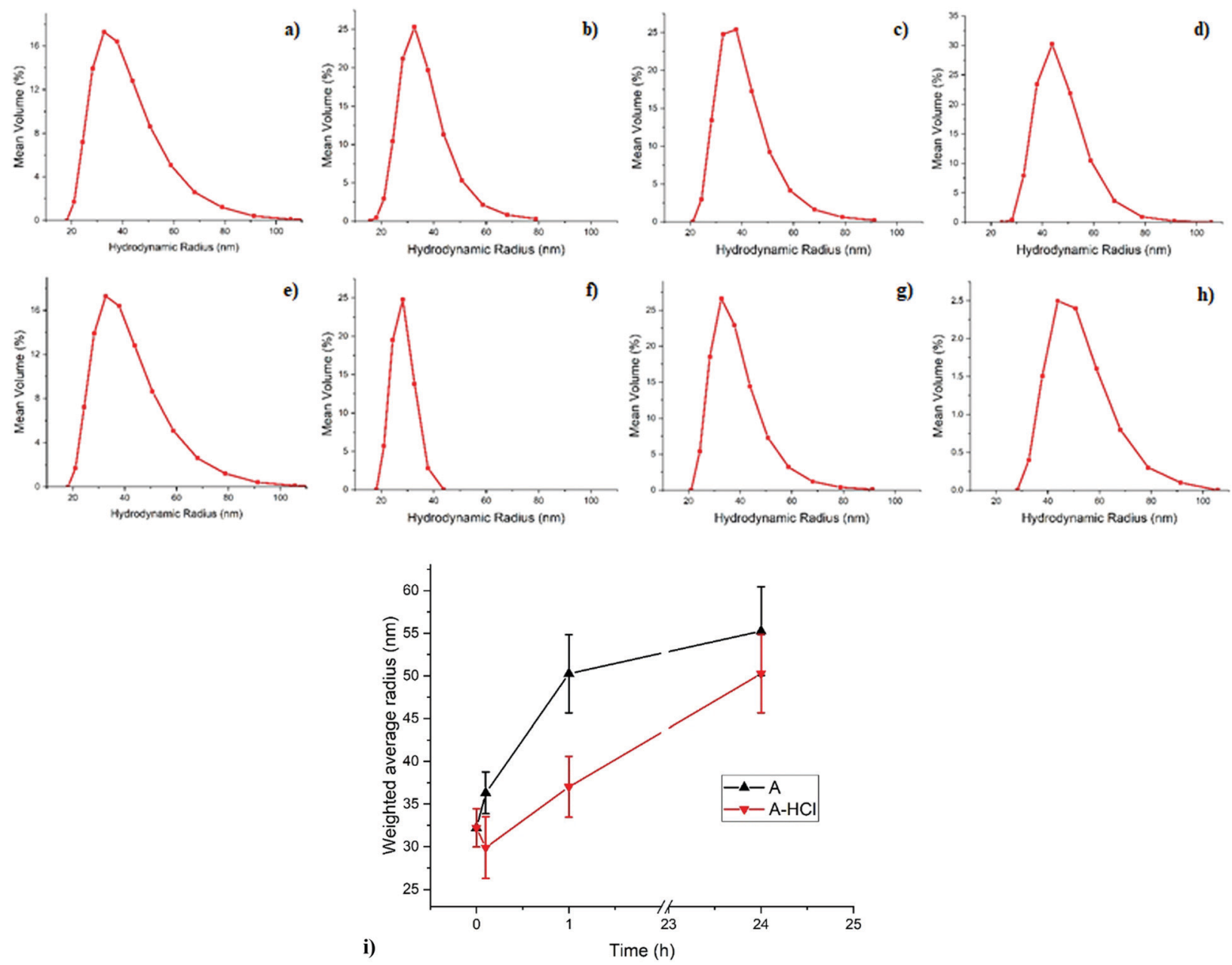

Fig. 1 DLS plots of samples A (a-d) and A-HCl (e-h). Sample A before reduction (a) and after $0.1 \mathrm{~h}$ (b), $1 \mathrm{~h}(\mathrm{c})$ and $24 \mathrm{~h}$ (d) of the reduction process. Sample A-HCl before reduction (e) and after $0.1 \mathrm{~h} \mathrm{(f),} 1 \mathrm{~h}(\mathrm{~g})$ and $24 \mathrm{~h}(\mathrm{~h}$ ) of the reduction process. In (i) are shown the weighted average radius obtained from the DLS measurements as function of time for sample $\mathrm{A}$ and $\mathrm{A}-\mathrm{HCl}$. The average diameter of the polymer micelle increases from $\sim 60$ to $\sim 110 \mathrm{~nm}$ after the reduction for both samples.

spectra shown in Fig. 2a for sample A contain a plasmon peak at $550 \mathrm{~nm}$ characteristic of $\mathrm{Au}^{0}$ clusters formed by the reduction of $\mathrm{HAuCl}_{4}{ }^{44}$ The increase in intensity with time suggests continuous oxidic Au species reduction and formation of Au NPs; interestingly the band does not change position suggesting continued growth of the Au nanoparticles without any dramatic
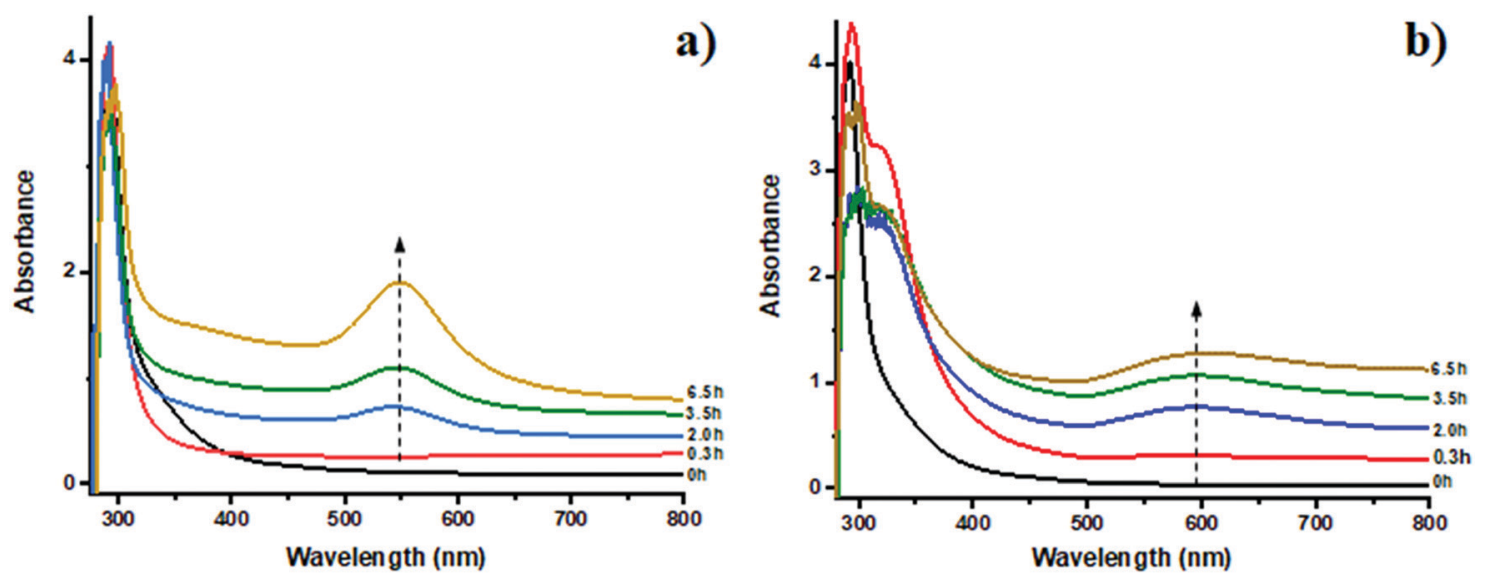

Fig. 2 UV-vis spectra of samples A (a) and A- $\mathrm{HCl}$ (b) during reduction. The dotted line, added as an eye guide, represents the maximum of the plasmon peak that is $550 \mathrm{~nm}$ for sample $A$ and $600 \mathrm{~nm}$ for sample A-HCl. ${ }^{44}$ 
change in size. In the case of sample A-HCl, the red shift of the plasmon peak from 550 to $600 \mathrm{~nm}$ in Fig. $2 \mathrm{~b}$ could be explained by nanoparticles aggregation possibly caused by the presence of "water pockets" induced by the addition of $\mathrm{HCl}$, by the unlikely possibility that particles larger than the micelles themselves ( $\gg 100 \mathrm{~nm}$ ) are forming or else by the presence of anisotropic particles. ${ }^{45}$ It can be also noted that characteristic plasmon band at $6.5 \mathrm{~h}$ in Fig. $2 \mathrm{~b}$ has lower intensity compared to spectrum at $3.5 \mathrm{~h}$. This could be possibly due to the precipitation of some of the already formed $\mathrm{Au}$ nanoparticles, causing a decrease in the amount of $\mathrm{Au}$ species which are observed by the beam. After $24 \mathrm{~h}$ of reduction since the micelles containing reduced Au NPs were observed to precipitate as can be seen in Fig. S1 (ESI $\dagger$ ) and hence no UV-vis data could be reported. Additional band located at $\sim 330 \mathrm{~nm}$ (Fig. 2b) decreases in intensity. This peak could be attributed to gold(III) chloride species that are slowly consumed during the reduction. ${ }^{46}$ To follow the changes in the gold clusters' size and oxidation state of the Au species during the reduction process a combination of XANES and EXAFS was utilised. ${ }^{10,27}$

\subsection{XAFS}

In situ XAFS was used to determine further the mechanism of the $\mathrm{Au}$ reduction and aggregation of the gold clusters. The results of the EXAFS fit for all four samples after 0 and $2.25 \mathrm{~h}$ of the reduction process are summarised in Table 2. Fig. 3 contains XANES spectra and EXAFS Fourier Transforms (FTs) for samples A and A-HCl. A reference $\mathrm{AuCl}_{3}$ solution which contains a strong white line at $11.920 \mathrm{keV}$ due to an electronic (dipole) transition from the $2 \mathrm{p}$ orbitals to the partially unoccupied $5 \mathrm{~d}$ orbitals is also shown in Fig. 3a and c. ${ }^{47}$ After the reductant is added there is a drop in the intensity in the white line (reduced transition probability) as a consequence of increased filling of the Au $5 \mathrm{~d}$ orbitals. This change is observed in conjunction with a slight shift of the edge position towards higher energies (from 11.197 to $11.199 \mathrm{keV}$ ). Both changes are consistent with the reduction of $\mathrm{Au}(\mathrm{III})$ to $\mathrm{Au}(\mathrm{I}) .{ }^{48}$ However, no further changes were observed in the XANES between 0 and $2.25 \mathrm{~h}$ suggesting the Au(I) species formed are stable.

The EXAFS data for sample A in Fig. 3b are clearly different to that of the reference compounds but do possess a peak at $\sim 2.24 \AA$ which can be assigned to a Au-Cl scattering contribution, although the reduced amplitude (when compared with $\mathrm{AuCl}_{4}$ in solution) suggests the presence of $\mathrm{Au}(\mathrm{I}) \mathrm{Cl}_{x}$ species (see eqn (4)). These values (and FTs) are similar to those observed previously by Salama et ll $^{49}$ in Na-Y zeolites and are consistent with the formation of $\mathrm{AuCl}$ species. $\mathrm{Au}(\mathrm{I})$ species in the form of a $\mathrm{Au}_{2} \mathrm{Cl}_{2}$ closed square dimer configuration previously reported by Hargittai et al. ${ }^{50}$ can be ruled out on the basis that the $\mathrm{Au}-\mathrm{Cl}$ distance reported there $(2.567 \AA)$ is too long. Interestingly, the Debye-Waller factors obtained for both samples are comparatively low suggesting low static/thermal disorder in the AuCl species. No sign of any metallic Au NPs were observed after $2.25 \mathrm{~h}$ of reaction (see Fig. $3 \mathrm{~b}$ ).

For sample $\mathrm{A}-\mathrm{HCl}$, the higher $\mathrm{Au}-\mathrm{Cl}$ coordination number $(>2)$ at the start of the reduction $(0 \mathrm{~h})$ suggests that initially the reduction process is slightly less advanced in comparison to the non-HCl samples. However, after $2.25 \mathrm{~h}$ the formation of $\mathrm{Au}$ NPs can be seen by the presence of the $\mathrm{Au}-\mathrm{Au}$ scattering contribution at $2.86 \AA$ in the second shell of the FT (Fig. 3d). Assuming an isotropic shape, one can estimate the size of the $\mathrm{Au}$ NPs from the $\mathrm{CN}_{\mathrm{Au}-\mathrm{Au}}$ value to be of the order of $\sim 2 \mathrm{~nm} .^{51}$ However, reduction is not complete at this stage as a clearly discernible, if low, $\mathrm{CN}$ value is obtained for the $\mathrm{Au}-\mathrm{Cl}$ first shell. XANES spectra also confirm the presence of metallic Au by the increase intensity of the feature located at $11.945 \mathrm{keV}$ which can be attributed to a multiple scattering path of metallic $\mathrm{Au}^{52}$ (Fig. 3c). However, the higher edge energy and the intense white

Table 2 EXAFS fit results for Au species with time/composition

\begin{tabular}{|c|c|c|c|c|c|c|c|c|c|}
\hline Sample & Time (h) & $\mathrm{CN}_{\mathrm{Au}-\mathrm{Cl}}$ & $R_{\mathrm{Au}-\mathrm{Cl}}(\AA)$ & $\sigma_{\mathrm{Au}-\mathrm{Cl}}^{2}\left(\AA^{-2}\right)$ & $\mathrm{CN}_{\mathrm{Au}-\mathrm{Au}}$ & $R_{\mathrm{Au}-\mathrm{Au}}(\AA)$ & $\sigma_{\mathrm{Au}-\mathrm{Au}}^{2}\left(\AA^{-2}\right)$ & $\Delta E$ & $R_{\text {factor }}$ \\
\hline $\mathrm{AuCl}_{4}$ in solution & - & $4.29 \pm 0.18$ & $2.280 \pm 0.003$ & $0.0020 \pm 0.0003$ & & & & $4.29 \pm 0.18$ & 0.007 \\
\hline \multirow[t]{2}{*}{$\mathrm{A}-\mathrm{HCl}$} & 0 & $2.9 \pm 0.4$ & $2.266 \pm 0.009$ & $0.0025 \pm 0.0013$ & - & - & - & $8.1 \pm 1.3$ & 0.03 \\
\hline & 2.25 & $0.7 \pm 0.4$ & $2.25 \pm 0.02$ & $0.0027 \pm 0.0018$ & $8.2 \pm 1.8$ & $2.86 \pm 0.01$ & $0.007 \pm 0.002$ & $6.9 \pm 1.3$ & 0.05 \\
\hline
\end{tabular}

$\mathrm{CN}$, the coordination number; $R$, the radial distance; $\sigma^{2}$, the Debye-Waller factor.
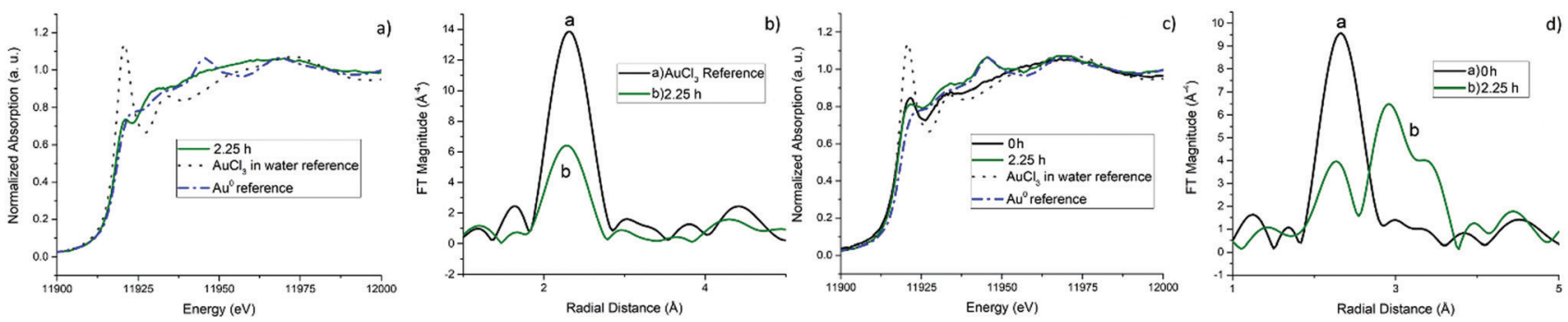

Fig. 3 XAFS spectra of sample A ( $a$ and $b$ ) at $2.25 \mathrm{~h}$ of reduction time and $\mathrm{A}-\mathrm{HCl}$ ( $\mathrm{c}$ and $\mathrm{d}$ ) at 0 and $2.25 \mathrm{~h}$ of reduction time. XANES spectra include $\mathrm{Au}{ }^{\circ}$ and $\mathrm{AuCl}_{3}$ references for sample A (a) and A- $\mathrm{HCl}$ (c). EXAFS Fourier Transform for sample A (b) and A- $\mathrm{HCl}$ (d). 

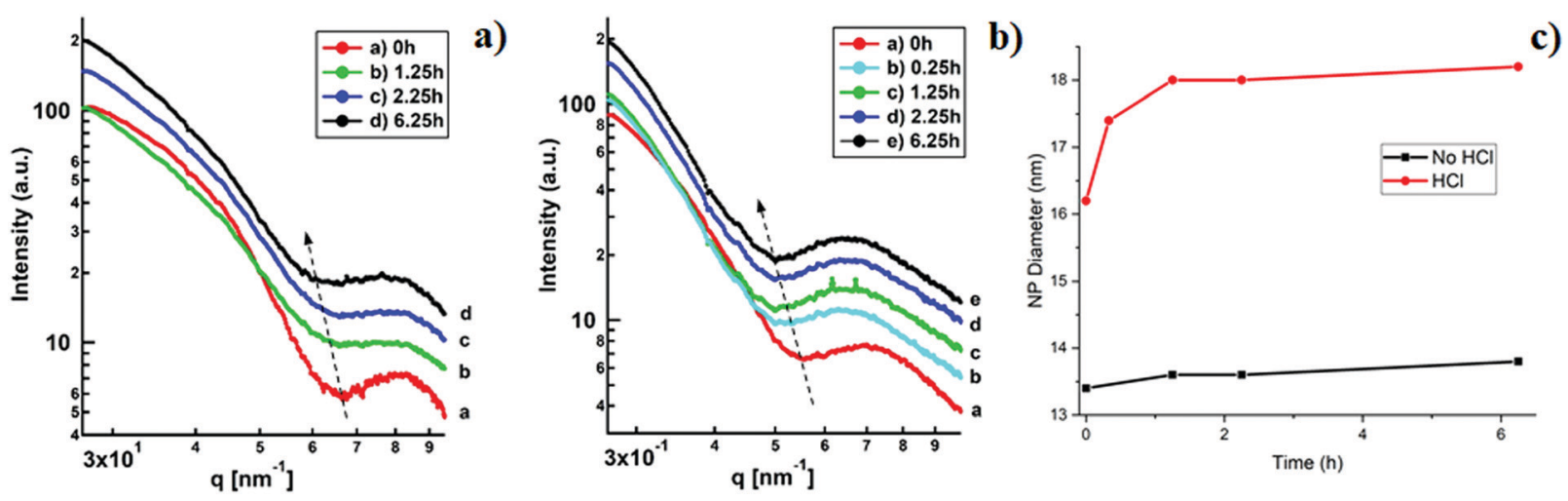

Fig. 4 Structural changes during reduction as followed by in situ SAXS. 1D SAXS profiles for the sample A (a), A- HCl (b). Evolution of the micelles core diameter with time for the samples $\mathrm{A}$ and $\mathrm{A}-\mathrm{HCl}$ (c).

line of sample $\mathrm{A}-\mathrm{HCl}$ compared to $\mathrm{Au}^{0}$ also suggest that $\mathrm{Au}(\mathrm{I}) \mathrm{Cl}_{x}$ species are still present after $2.25 \mathrm{~h}$.

\subsection{SAXS}

In order to investigate the structural transformation, in particular in terms of size and polydispersity, of the NPs formed in the micelle's core during the reduction process in situ SAXS studies were conducted. 1D SAXS profiles for samples A and A-HCl during the reduction process are shown in Fig. 4. According to the fit (for the details see Experimental section and Fig. S2 and S3) there is a slight increase in the scattering component size from 13.4 to $13.8 \mathrm{~nm}$ (Fig. 4a, c and Fig. S2, ESI $\dagger$ ) for sample A after $6.25 \mathrm{~h}$ of reduction. For sample A-HCl a shift of the scattering component towards smaller $q$-values can be seen which corresponds to sizes from 16.2 to $18.2 \mathrm{~nm}$ depending on reduction time, with $1.25 \mathrm{~h}$ being the time when the size stabilizes (Fig. 4b, c and Fig. S3, ESI†).

The polydispersity of the system ${ }^{53}$ obtained from the fit results is relatively low $(\leq 13 \%)$. The calculated value of polydispersity is stable throughout the NP formation process (see Fig. S2 and S3, ESI + ), indicating that as the NPs grow the homogeneity of the system is not affected.

The in situ SAXS fit results summarised in Fig. 4c show a growth of the NPs for both samples during the reduction process that is in a good agreement with the DLS data (Fig. 1) whereas the difference in size obtained for sample A ( $\sim 14 \mathrm{~nm}$ from SAXS, $\sim 60 \mathrm{~nm}$ from
DLS) is due to the PS shell which is not detected by the SAXS due the low electron density contrast between toluene and polystyrene (Fig. S4a, ESI $\dagger$ ). The increase of the P2VP core size from $\sim 16$ to $\sim 18 \mathrm{~nm}$ for sample A-HCL, when $\mathrm{HCl}$ is included into the solution can be attributed to an increment in the speed of NPs' growth. This was also suggested from the XAFS analysis, indicating that $\mathrm{HCl}$ acts as a catalyst for the reduction of the Au species.

\subsection{TEM}

TEM has been used to characterize the morphology (e.g. shape and size) of the polymer micelles before and after adding reductant. As an example, Fig. 5a and b shows the PS- $b$-P2VP micelles loaded initially only with $\mathrm{HAuCl}_{4}$ for sample A-HCl. It can be seen that the metal ions are preferentially located in the micelle cores (Fig. 5a and b). Although, for sample A-HCl, the gold appears as sub-nanometre clusters inside the core of the micelle, which we propose is caused by the reduction of the precursor caused by the electron beam (Fig. 5b). The loaded micelles' core size for sample A-HCl is $\sim 11 \mathrm{~nm}$ (Fig. $5 \mathrm{~b}$ ). The fact that the micelle's core size observed in TEM compares well with the SAXS data further supports the assumption that $\mathrm{Au}$ species are confined and selfassemble from within the polymer micelle core.

After $p$-tosyl hydrazide is added to the micelle-containing solution, reduction of $\mathrm{Au}$ ions occurs and leads to the formation of nanoparticles, as shown for example in Fig. $5 \mathrm{c}$ and $d$
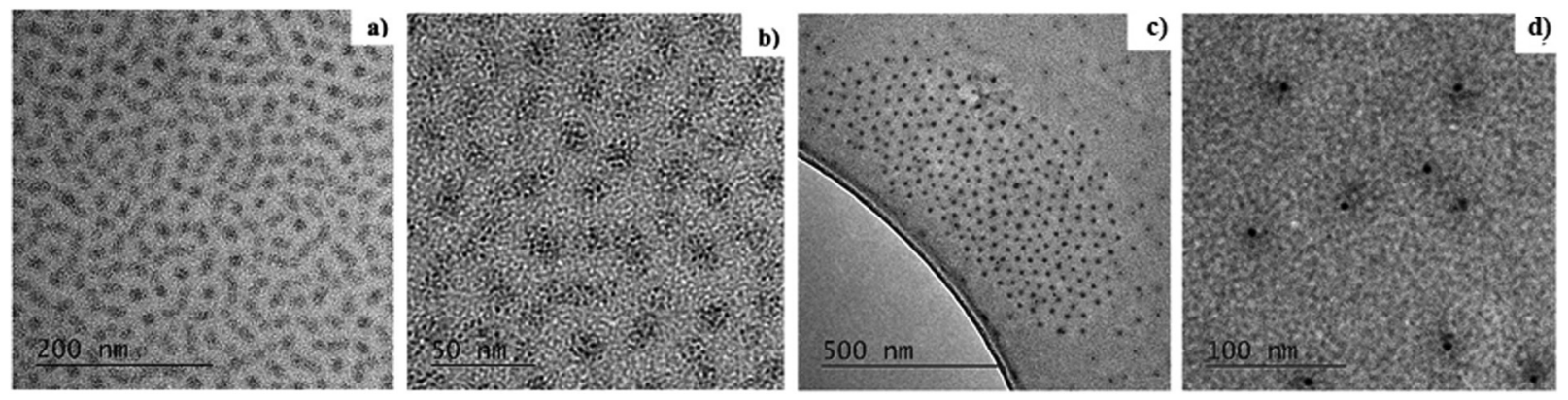

Fig. 5 TEM micrographs of sample A- $\mathrm{HCl}$ before ( $\mathrm{a}$ and $\mathrm{b}$ ) and after reduction ( $\mathrm{c}$ and d). Polymer A loaded with $\mathrm{HAuCl}_{4}$ before the addition of reductant (a) and higher magnification image (b). Average micelle core size is $\sim 11 \mathrm{~nm}$. Sample $\mathrm{A}-\mathrm{HCl}$ after addition of reducing agent and $\mathrm{HCl}$ (c) and higher magnification image (d) with the average NP size of $4.5 \pm 0.8 \mathrm{~nm}$. 
for the reduced sample A- $\mathrm{HCl}$ after $24 \mathrm{~h}$. The calculated NP size from the TEM data is $4.5 \pm 0.8 \mathrm{~nm}$ that is considerably smaller than the value of $\sim 11 \mathrm{~nm}$ for the micelle core of sample A-HCl before the reduction.

\subsection{Rationalisation for self-assembly process}

From the results obtained by the combination of time-resolved DLS, UV-vis, XAFS, SAXS and TEM it was possible to propose a model for the self-assembly of the homogeneous metal nanoparticles in the inverse micelles system shown in Scheme 1, that can be delineated into the following steps:

- an initial fast reduction of $\left[\mathrm{AuCl}_{4}\right]^{-}$to $\mathrm{Au}(\mathrm{I}) \mathrm{Cl}_{x}$ species;

- further slow reduction of the $\mathrm{Au}(\mathrm{I})$ and formation of $\mathrm{Au}(0)$ clusters;

- agglomeration of the small clusters to form nanoparticles confined inside the core of the micelle.

The rational for our scheme is given as follows. Firstly, according to TEM data (Fig. 5), the micelle core is loaded with the gold precursor at the beginning of synthesis (stage 1 in Scheme 1). This in combination with the XAFS data dominated by $\mathrm{Au}-\mathrm{Cl}$ species leads us to conclude that Au must be initially homogeneously dispersed all over the micelle cores as a complex between the nitrogen from pyridine and $\left[\mathrm{AuCl}_{4}\right]^{-}$, in a similar way to that previously observed for iron and molybdenum precursors. ${ }^{54}$ After the reductant is added (stage 2 in Scheme 1) the $\left[\mathrm{AuCl}_{4}\right]^{-}$ions reduce almost immediately to $\mathrm{Au}(\mathrm{I})$ species, namely $\left[\mathrm{AuCl}_{2}\right]^{-}$, this again concluded mainly on the basis of the XAFS results (Table 2 and XANES data shown in Fig. 3). Furthermore, we observe no immediate change in the core diameter from the SAXS data with reaction time (Fig. 4). The core diameter obtained from the fit of the SAXS data, $13.4 \mathrm{~nm}$ for sample A and $16.2 \mathrm{~nm}$ for sample A-HCl (Fig. S2 and S3, ESI $\dagger$ ), in addition to the TEM data, suggests that the $\left[\mathrm{AuCl}_{2}\right]^{-}$species are well dispersed within the micelle core; this we conclude based on the good agreement obtained for SAXS and TEM measurements for the size of the micelle core when filled with Au species (Scheme 1).

After $2.25 \mathrm{~h}$ (stage 3 in Scheme 1) the formation of $\mathrm{Au}^{0}$ species can be seen for sample A-HCl (Fig. 3d), likely comprised of small clusters based on the low EXAFS-derived values of $\mathrm{CN}_{\mathrm{Au}-\mathrm{Au}}$ shown in Table 2. An increase of the NP diameter at this time during the reduction process measured using SAXS indicates self-assembly of gold clusters into the NPs (Fig. 4). The swelling of the micelle core continues (Fig. 4c) until Au reduction is complete. Interestingly for sample A whereas XAFS does not show any presence of $\mathrm{Au}^{0}$ species, the UV-vis data display a, albeit very small, plasmon peak (Fig. 2a). This could be attributed by the large amount of $\mathrm{Au}(\mathrm{I})$ species which dominate the XAFS spectra, whereas UV-vis is only sensible to the few $\mathrm{Au}^{0}$ species present in solution.

Further agglomeration of the gold clusters to form NPs (stage 4 in Scheme 1) was confirmed by the UV-vis (Fig. 2) and TEM data (Fig. 5c and d), the latter showing that once the reduction process is completed, the formed NPs are positioned at the centre of the micelles. Moreover, the nanoparticles show a defined spherical shape, that is clearly different from the subnanometric gold cluster formed due to electron beam exposure (Fig. 5a and b). We note however that the DLS data revealed that the whole micelle, and not just the core, is affected during the reduction of the gold precursor to the metal NP (Fig. 1).

\subsection{The role of $\mathrm{HCl}$ in the self-assembly process}

The XAFS, UV-vis and SAXS data suggest that the presence of $\mathrm{HCl}$ catalyses the reduction process of $\mathrm{Au}(\mathrm{I})$ to $\mathrm{Au}^{0}$ and its agglomeration.
1)

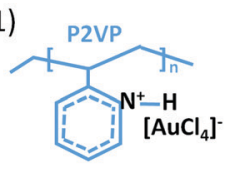

2)

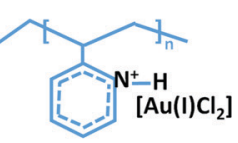

3)

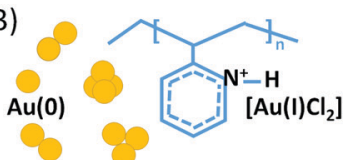

4)

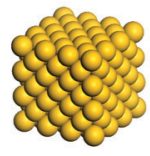

AGGLOMERATION Au Nanoparticle

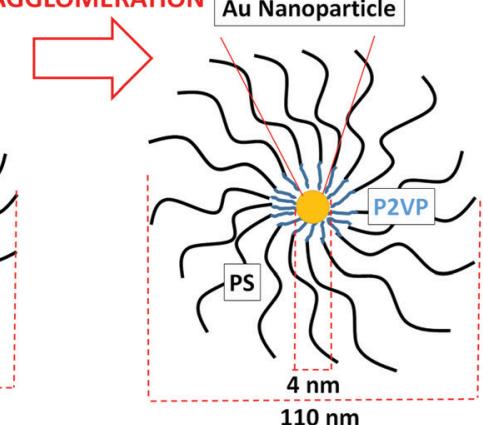

time

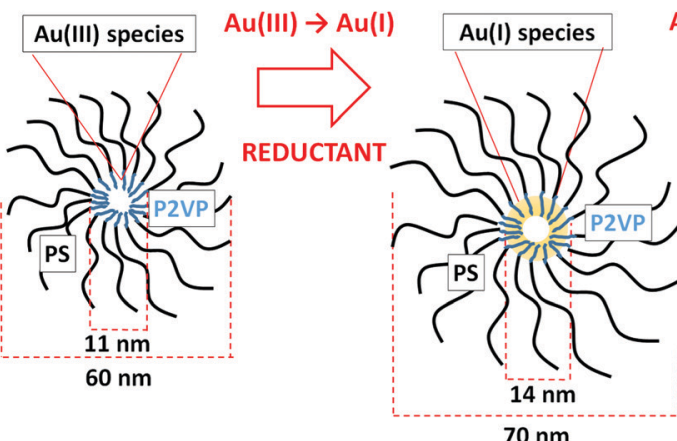

$<0.1$ h $\sim 2.25 \mathrm{~h}$

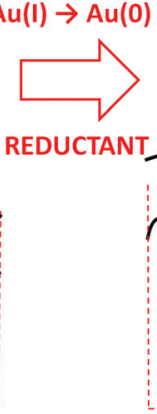

$>6 \mathrm{~h}$ (or $\mathrm{HCl})$

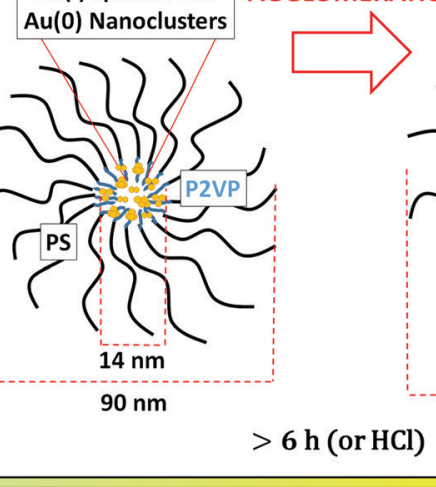

XAFS, SAXS, DLS, TEM, UV-Vis
TEM, UV-Vis

Scheme 1 Proposed schematic model of the Au nanoparticle formation during the polymer inverse micelle synthesis, including the time scale and techniques used to identify each stage. (1) the $\left[\mathrm{AuCl}_{4}\right]^{-}$ions attach to the pyridine group; (2) the $\left[\mathrm{AuCl}_{4}\right]^{-}$ions get reduced to $\mathrm{Au}(\mathrm{I})$ species; (3) the $\mathrm{Au}(\mathrm{I})$ species are reduced to form $A u(0)$ species that start to form Au nanoclusters; (4) resulted Au nanoparticle encapsulated in the PS-P2VP micelle. Characteristic sizes are shown using an example of sample A. The addition of $\mathrm{HCl}$ increase the rate of formation of Au clusters. 
We rationalise that $\mathrm{HCl}$ helps with the in situ formation of hydrazine through the following reaction sequence:

$\mathrm{NH}_{2}-\mathrm{NH}$ - $p$-toluenesulfonyl $+\mathrm{HCl} \leftrightarrow \mathrm{NH}_{2} \mathrm{NH}_{2}+\mathrm{Cl}-p$-toluenesulfonyl

which will then act as a reducing agent according to the following equations

$$
\begin{aligned}
2 \mathrm{H}\left[\mathrm{AuCl}_{4}\right]+\mathrm{N}_{2} \mathrm{H}_{4} & \rightarrow \mathrm{N}_{2}+6 \mathrm{HCl}+2 \mathrm{AuCl} \\
4 \mathrm{AuCl}+\mathrm{N}_{2} \mathrm{H}_{4} & \rightarrow \mathrm{N}_{2}+4 \mathrm{HCl}+4 \mathrm{Au}
\end{aligned}
$$

that can be summarised as:

$$
4 \mathrm{H}\left[\mathrm{AuCl}_{4}\right]+3 \mathrm{~N}_{2} \mathrm{H}_{4} \rightarrow 4 \mathrm{Au}+3 \mathrm{~N}_{2}+16 \mathrm{HCl}
$$

One can conclude that the rate of $\mathrm{N}_{2} \mathrm{H}_{4}$ production (eqn (7)) increases and becomes autocatalytic due to the formation of $\mathrm{HCl}$ as a by-product of Au reduction (eqn (8) and (9)). Therefore, it is plausible to assume that $\mathrm{HCl}$ added at the start accelerates the formation of $\mathrm{N}_{2} \mathrm{H}_{4}$ and therefore increases the rate and the extent of reduction. The amplified $\mathrm{N}_{2} \mathrm{H}_{4}$ production seems to lead to, in addition to fast $\mathrm{Au}(\mathrm{I})$ reduction, nucleation of $\mathrm{Au}$ clusters (see UV-vis data in Fig. 2) although the final Au NPs possess similar characteristics to those obtained from the samples without HCl. This suggests that the final step, agglomeration, was given enough time to occur in both cases (with and without $\mathrm{HCl}$ ) and that this step is important for realising uniformity in the particle size. New insights into the selfassembly mechanism and the impact of $\mathrm{HCl}$ allows to utilise different reaction times/conditions to change the properties of the final nanoparticles i.e. stopping the reaction earlier may produce the 'raspberry type' Au NPs or else to help to choose the right time to incorporate different species, for example metal ions, to produce either bimetallic nanoclusters/alloys (a second metal precursor is added during nucleation process) or core shell NPs (a second metal is added to the already formed nanoparticle). ${ }^{51,54}$

The low polydispersity values calculated from the SAXS data are consistent with the presence of micelles containing evenly loaded and highly dispersed Au ions attached as molecular species to the pyridine groups of the P2VP chain. We propose that this interaction is important as it slows the reduction of $\mathrm{Au}(\mathrm{I})$ to $\mathrm{Au}^{0}$ and agglomeration process, compared to more commonly used Au NP synthesis procedures such as the Turkevich method. ${ }^{10,55}$ This retardation then seems to realise a more controlled Au NP nucleation and growth process resulting in a much narrower particle size distribution. This also demonstrates the protective effect of the polymer micelles, preventing undesired coalescence of metal NPs. Our results can be interpreted as being more widely reflective of how Au NP assembly occurs in the presence of species that can coordinate and/or complex with Au species (i.e. block co-polymers, capping agents such as PVP, ${ }^{56} \mathrm{PVA}^{57}$ etc.). For example, Sakai et al. using block PEO-PPO-PEO co-polymers observed simultaneously the reduction of $\left[\mathrm{AuCl}_{4}\right]^{-}$precursor to $\mathrm{Au}^{0}$, formation of Au clusters and finally the growth of the NPs. ${ }^{19}$ Due to the comparative slowness the inverse polymer micelle encapsulation method allows to separate different stages of Au NP growth and to follow the formation of homogeneous particles step by step. Moreover timeresolved X-ray techniques help to unequivocally identify the presence of a stable $\mathrm{Au}(\mathrm{I})$ species as intermediates in the formation process. Indeed, such $\mathrm{Au}(\mathrm{I})$ species have been reported only for the short time ( $\leq 30 \mathrm{~min}$ ) during Au NP self-assembly, ${ }^{58,59}$ partially due to the fast disproportionation to $\mathrm{Au}^{0}$ and $\mathrm{Au}(\mathrm{III})$.

\subsection{Effects of X-ray irradiation on stability of Au species}

Shown in Fig. S4b (ESI $\dagger$ ) are two XANES spectra acquired in succession for sample A, 5 min after addition of the reductant. The similarity of the two XANES spectra are testament of the stability of the samples in the X-ray beam and indicate that the mixed $\mathrm{Au}$ metal/ionic environments observed are a direct consequence of a slow reduction process.

\section{Conclusions}

Although the formation of Au NPs within polymer-encapsulated inverse micelles is a dynamic process, by obtaining multi-technique 'snap-shots' during this process, the key stages associated with their self-assembly could be identified. This is the first study, to the best of our knowledge, where the combination of SAXS/EXAFS methods and slow reducing agent allowed to obtain the comprehensive pathway on the mechanism of gold NPs formation. We speculate that whereas this mechanism has been explored for the inverse micelle method, it is highly likely that the formation process would be similar if not identical when using similar synthetic methods i.e. those which employ polymers or liquid crystals as capping agents, or complexes, etc. Importantly, when considering the application of such bottom-up synthetic procedures the detailed insight obtained here concerning the rate and mechanism of Au NP formation allows us to identify the variables, i.e. time or presence of additional reagents, that can be altered to aid production or else to tune the nanoparticle' properties, i.e. composition and morphology, thus allowing a better control over NP size and particle size distribution and ultimately to a more optimal performance in their intended application.

\section{Abbreviations}

NP Nanoparticle

SAXS Small-angle X-ray scattering

XAFS X-ray absorption fine structure

\section{Conflicts of interest}

There are no conflicts to declare.

\section{Acknowledgements}

The authors would like to thank the EPSRC for funding (grant EP/K007467/1 \& EP/L015862/1). The authors also thank NWO for part sponsorship of a studentship for JH and for DUBBLE@ESRF for the (in-house) beamtime. 


\section{References}

1 P. Ghosh, G. Han, M. De, C. K. Kim and V. M. Rotello, Gold nanoparticles in delivery applications, Adv. Drug Delivery Rev., 2008, 60, 1307-1315.

2 F. Tao and J. Spivey, Metal Nanoparticles for Catalysis, The Royal Society of Chemistry, Cambridge, 2014.

3 R. Elghanian, J. J. Storhoff, R. C. Mucic, R. L. Letsinger and C. A. Mirkin, Selective Colorimetric Detection of Polynucleotides Based on the Distance-Dependent Optical Properties of Gold Nanoparticles, Science, 1997, 277, 1078LP-1081LP.

4 M. Moritz and M. Geszke-Moritz, The newest achievements in synthesis, immobilization and practical applications of antibacterial nanoparticles, Chem. Eng. J., 2013, 228, 596-613.

5 G. Cao, Nanastructures and Nanomaterials - Synthesis, Properties and Applications, Imperial College Press, London, 2004, vol. 2.

6 A. S. Edelstein and R. C. Cammarata, Nanomaterials: synthesis, properties, and applications, Taylor \& Francis, Abingdon, 1998.

7 P. A. Schaal, A. Besmehn, E. Maynicke, M. Noyong, B. Beschoten and U. Simon, Electrically Conducting Nanopatterns Formed by Chemical e-Beam Lithography via Gold Nanoparticle Seeds, Langmuir, 2012, 28, 2448-2454.

8 A. Longo, P. Calandra, M. P. Casaletto, C. Giordano, A. M. Venezia and V. T. Liveri, Synthesis and physico-chemical characterization of gold nanoparticles softly coated by AOT, Mater. Chem. Phys., 2006, 96, 66-72.

9 W. Karim, A. Kleibert, U. Hartfelder, A. Balan, J. Gobrecht, J. A. van Bokhoven and Y. Ekinci, Size-dependent redox behavior of iron observed by in-situ single nanoparticle spectro-microscopy on well-defined model systems, Sci. Rep., 2016, 6, 18818.

10 J. Polte, T. T. Ahner, F. Delissen, S. Sokolov, F. Emmerling, A. F. Thünemann and R. Kraehnert, Mechanism of Gold Nanoparticle Formation in the Classical Citrate Synthesis Method Derived from Coupled In Situ XANES and SAXS Evaluation, J. Am. Chem. Soc., 2010, 132, 1296-1301.

11 B. Yin, H. Ma, S. Wang and S. Chen, Electrochemical Synthesis of Silver Nanoparticles under Protection of Poly(N-vinylpyrrolidone), J. Phys. Chem. B, 2003, 107, 8898-8904.

12 A. Nemamcha, J.-L. Rehspringer and D. Khatmi, Synthesis of Palladium Nanoparticles by Sonochemical Reduction of Palladium(II) Nitrate in Aqueous Solution, J. Phys. Chem. B, 2006, 110, 383-387.

13 H. Zhang, W. Wang, M. Akinc, S. Mallapragada, A. Travesset, D. Vaknin, S. Mallapragada, D. Vaknin, T. Xu and A. P. Alivisatos, Assembling and ordering polymer-grafted nanoparticles in three dimensions, Nanoscale, 2017, 9, 8710-8715.

14 M. C. Saint-Lager, I. Laoufi, A. Bailly, O. Robach, S. Garaudee and P. Dolle, Catalytic properties of supported gold nanoparticles: new insights into the size-activity relationship gained from in operando measurements, Faraday Discuss., 2011, 152, 253-265.

15 I. Laoufi, M. C. Saint-Lager, R. Lazzari, J. Jupille, O. Robach, S. Garaudée, G. Cabailh, P. Dolle, H. Cruguel and A. Bailly, Size and catalytic activity of supported gold nanoparticles: An in operando study during CO oxidation, J. Phys. Chem. C, 2011, 115, 4673-4679.
16 A. Kabelitz, A. Guilherme, M. Joester, U. Reinholz, M. Radtke, R. Bienert, K. Schulz, R. Schmack, R. Kraehnert and F. Emmerling, Time-resolved in situ studies on the formation mechanism of iron oxide nanoparticles using combined fastXANES and SAXS, CrystEngComm, 2015, 17, 8463-8470.

17 J. Polte, X. Tuaev, M. Wuithschick, A. Fischer, A. F. Thuenemann, K. Rademann, R. Kraehnert and F. Emmerling, Formation Mechanism of Colloidal Silver Nanoparticles: Analogies and Differences to the Growth of Gold Nanoparticles, ACS Nano, 2012, 7, 5791-5802.

18 N. T. K. Thanh, N. Maclean and S. Mahiddine, Mechanisms of Nucleation and Growth of Nanoparticles in Solution, Chem. Rev., 2014, 114, 7610-7630.

19 T. Sakai and P. Alexandridis, Single-step synthesis and stabilization of metal nanoparticles in aqueous pluronic block copolymer solutions at ambient temperature, Langmuir, 2004, 20, 8426-8430.

20 J. P. Spatz, S. Mössmer, C. Hartmann, M. Möller, T. Herzog, M. Krieger, H. G. Boyen, P. Ziemann and B. Kabius, Ordered deposition of inorganic clusters from micellar block copolymer films, Langmuir, 2000, 16, 407-415.

21 D. James Martin, D. Decarolis, Y. I. Odarchenko, J. J. Herbert, T. Arnold, J. Rawle, C. Nicklin, H.-G. Boyen and A. M. Beale, Reversible restructuring of supported $\mathrm{Au}$ nanoparticles during butadiene hydrogenation revealed by operando GISAXS/ GIWAXS, Chem. Commun., 2017, 53, 5159-5162.

22 D. J. Martin, D. Decarolis, R. Tucoulou, G. Martínez-Criado and A. M. Beale, Towards the interrogation of the behaviour of a single nanoparticle under realistic catalytic reaction conditions, Catal., Struct. React., 2017, 3, 63-70.

23 U. Wiedwald, L. Han, J. Biskupek, U. Kaiser and P. Ziemann, Preparation and characterization of supported magnetic nanoparticles prepared by reverse micelles, Beilstein J. Nanotechnol., 2010, 1, 24-47.

24 B. R. Cuenya, Metal nanoparticle catalysts beginning to shape-up, Acc. Chem. Res., 2013, 46, 1682-1691.

25 H. Mistry, R. Reske, Z. Zeng, Z.-J. Zhao, J. Greeley, P. Strasser and B. R. Cuenya, Exceptional Size-Dependent Activity Enhancement in the Electroreduction of $\mathrm{CO} 2$ over $\mathrm{Au}$ Nanoparticles, J. Am. Chem. Soc., 2014, 136, 16473-16476.

26 P. Calandra, C. Giordano, A. Longo and V. T. Liveri, Physicochemical investigation of surfactant-coated gold nanoparticles synthesized in the confined space of dry reversed micelles, Mater. Chem. Phys., 2006, 98, 494-499.

27 M. Harada and Y. Kamigaito, Nucleation and Aggregative Growth Process of Platinum Nanoparticles Studied by in Situ Quick XAFS Spectroscopy, Langmuir, 2012, 28, 2415-2428.

28 G. F. Tierney, D. Decarolis, N. Abdullah, S. M. Rogers, S. Hayama, M. Briceno de Gutierrez, A. Villa, C. R. A. Catlow, P. Collier, N. Dimitratos and P. P. Wells, Extracting structural information of Au colloids at ultra-dilute concentrations: identification of growth during nanoparticle immobilization, Nanoscale Adv., 2019, 1, 2546-2552.

29 Z. Zhang and Y. Wu, Investigation of the NaBH4-Induced Aggregation of Au Nanoparticles, Langmuir, 2010, 26, 9214-9223. 
30 L. Bronstein, M. Antonietti and P. Valetsky, Nanoparticles and Nanostructured Films, Wiley-VCH Verlag GmbH, 1998, pp. 145-171.

31 J.-M. Yun, J.-S. Yeo, J. Kim, H.-G. Jeong, D.-Y. Kim, Y.-J. Noh, S.-S. Kim, B.-C. Ku and S.-I. Na, Solution-Processable Reduced Graphene Oxide as a Novel Alternative to PEDOT:PSS Hole Transport Layers for Highly Efficient and Stable Polymer Solar Cells, Adv. Mater., 2011, 23, 4923-4928.

32 S. R. Kline, Reduction and analysis of SANS and USANS data using IGOR Pro, J. Appl. Crystallogr., 2006, 39, 895-900.

33 M. Kotlarchyk, R. B. Stephens and J. S. Huang, Study of Schultz distribution to model polydispersity of microemulsion droplets, J. Phys. Chem., 1988, 92, 1533-1538.

34 O. Glatter and O. Kratky, Small Angle X-ray Scattering, Academic Press, London, 1982.

35 B. Ravel and M. Newville, Athena, artemis, hephaestus: data analysis for $\mathrm{x}$-ray absorption spectroscopy using IFEFFIT, J. Synchrotron Radiat., 2005, 12, 537.

36 M. Newville, IFEFFIT: interactive XAFS analysis and FEFF fitting, J. Synchrotron Radiat., 2001, 8, 322.

37 H. B. Bohidar and M. Behboudnia, Characterization of reverse micelles by dynamic light scattering, Colloids Surf., A, 2001, 178, 313-323.

38 R. D. Falcone, J. J. Silber and N. M. Correa, What are the factors that control non-aqueous/AOT/n-heptane reverse micelle sizes? A dynamic light scattering study, Phys. Chem. Chem. Phys., 2009, 11, 11096.

39 D. Voulgaris, C. Tsitsilianis, V. Grayer, F. J. Esselink and G. Hadziioannou, Amphiphile micelles formed by polystyrene/ poly(2-vinyl pyridine) heteroarm star copolymers in toluene, Polymer, 1999, 40, 5879-5889.

40 B. N. Khlebtsov and N. G. Khlebtsov, On the measurement of gold nanoparticle sizes by the dynamic light scattering method, Colloid J., 2011, 73, 118-127.

41 C. M. Hoo, N. Starostin, P. West and M. L. Mecartney, A comparison of atomic force microscopy (AFM) and dynamic light scattering (DLS) methods to characterize nanoparticle size distributions, J. Nanopart. Res., 2008, 10, 89-96.

42 S. Mössmer, J. P. Spatz, M. Möller, T. Aberle, J. J. Schmidt, W. Burchard, S. Moessner, J. P. Spatz, M. Moeller, T. Aberle, J. J. Schmidt, W. Burchard, S. Mössmer, J. P. Spatz, M. Möller, T. Aberle, J. J. Schmidt, W. Burchard, S. Moessner, J. P. Spatz, M. Moeller, T. Aberle, J. J. Schmidt, W. Burchard, S. Mössmer, J. P. Spatz, M. Möller, T. Aberle, J. J. Schmidt, W. Burchard, S. Moessner, J. P. Spatz, M. Moeller, T. Aberle, J. J. Schmidt and W. Burchard, Solution Behavior of Poly(styrene)- b lock -poly(2vinylpyridine) Micelles Containing Gold Nanoparticles, Macromolecules, 2000, 33, 4791-4798.

43 J. P. Spatz, S. Sheiko and M. Möller, Ion-stabilized block copolymer micelles: film formation and intermicellar interaction, Macromolecules, 1996, 29, 3220-3226.

44 S. Link and M. A. El-Sayed, Size and Temperature Dependence of the Plasmon Absorption of Colloidal Gold Nanoparticles, J. Phys. Chem. B, 1999, 103, 4212-4217.

45 W. Haiss, N. T. K. Thanh, J. Aveyard and D. G. Fernig, Determination of Size and Concentration of Gold
Nanoparticles from UV-vis Spectra, Anal. Chem., 2007, 79, 4215-4221.

46 P. Kwolek and M. Wojnicki, The kinetic study of photoreduction of tetrachloroaurate acid by methanol in acidic media, J. Photochem. Photobiol., A, 2014, 286, 47-54.

47 P. Zhang and T. K. Sham, Tuning the electronic behavior of Au nanoparticles with capping molecules, Appl. Phys. Lett., 2002, 81, 736-738.

48 G. S. Pokrovski, B. R. Tagirov, J. Schott, E. F. Bazarkina, J.-L. Hazemann and O. Proux, An in situ X-ray absorption spectroscopy study of gold-chloride complexing in hydrothermal fluids, Chem. Geol., 2009, 259, 17-29.

49 T. M. Salama, T. Shido, R. Ohnishi and M. Ichikawa, EXAFS/ XANES, XRD, and UV-vis Characterization of Intrazeolitic Gold(I) Prepared by Monolayer Dispersion of AuCl3 inside Na-Y Zeolite, J. Phys. Chem., 1996, 100, 3688-3694.

50 M. Hargittai, A. Schulz, B. Réffy and M. Kolonits, Molecular Structure, Bonding, and Jahn-Teller Effect in Gold Chlorides: Quantum Chemical Study of AuCl3, Au2Cl6, AuCl4-, AuCl, and $\mathrm{Au} 2 \mathrm{Cl} 2$ and Electron Diffraction Study of Au2Cl6, J. Am. Chem. Soc., 2001, 123, 1449-1458.

51 A. M. Beale and B. M. Weckhuysen, EXAFS as a tool to interrogate the size and shape of mono and bimetallic catalyst nanoparticles, Phys. Chem. Chem. Phys., 2010, 12, 5562-5574.

52 S. N. Reifsnyder, H. H. Lamb and N. Carolina, Characterization of Silica-Supported Pd-Au Clusters by X-ray Absorption Spectroscopy, J. Phys. Chem. B, 1999, 2, 321-329.

53 R. Hilfiker, H.-F. Eicke, W. Sager, C. Steeb, U. Hofmeier and R. Gehrke, Form and Structure Factors of Water/AOT/Oil Microemulsions from Synchrotron SAXS, Ber. Bunsen-Ges., 1990, 94, 677-683.

54 A. Riskin, A. M. Beale, H.-G. Boyen, A. Vantomme, A. Hardy and M. K. Van Bael, The use of XAFS to determine the nature of interaction of iron and molybdenum metal salts within PS- $b$-P2VP micelles, Phys. Chem. Chem. Phys., 2013, 15, 1675-1681.

55 J. Kimling, M. Maier, B. Okenve, V. Kotaidis, H. Ballot and A. Plech, Turkevich Method for Gold Nanoparticle Synthesis Revisited, J. Phys. Chem. B, 2006, 110, 15700-15707.

56 S. M. Rogers, C. R. A. Catlow, C. E. Chan-Thaw, D. Gianolio, E. K. Gibson, A. L. Gould, N. Jian, A. J. Logsdail, R. E. Palmer, L. Prati, N. Dimitratos, A. Villa and P. P. Wells, Tailoring Gold Nanoparticle Characteristics and the Impact on AqueousPhase Oxidation of Glycerol, ACS Catal., 2015, 5, 4377-4384.

57 H. Shi, N. Xu, D. Zhao and B.-Q. Xu, Immobilized PVAstabilized gold nanoparticles on silica show an unusual selectivity in the hydrogenation of cinnamaldehyde, Catal. Commun., 2008, 9, 1949-1954.

58 B. Abécassis, F. Testard, Q. Kong, B. Francois and O. Spalla, Influence of Monomer Feeding on a Fast Gold Nanoparticles Synthesis: Time-Resolved XANES and SAXS Experiments, Langmuir, 2010, 26, 13847-13854.

59 F. Hubert, F. Testard, A. Thill, Q. Kong, O. Tache and O. Spalla, Growth and Overgrowth of Concentrated Gold Nanorods: Time Resolved SAXS and XANES, Cryst. Growth Des., 2012, 12, 1548-1555. 